\title{
Optimization in high-precision glass forming
}

Sellier, M.

Schott AG, Hattenbergstrasse 10, 55122 Mainz, Germany

mathieu.sellier@itwm.fhg.de

\begin{abstract}
Summary. The question of interest in the present study is the inverse problem for high precision glass forming, i.e. 'How to design the mould and the temperature regime so that at the very end of the forming process we will get at room temperature a prescribed glass geometry with a precision in the order of the Micron?' The aim is to eliminate from the manufacturing process the costly and time-consuming postprocessing when the final shape does not conform precisely to the desired one.
\end{abstract}

Key words: glass forming, stress/structural relaxation, optimization

\section{Description of the forward problem}

The present study focuses on the cooling stage of the glass forming process and provides a method based on computer-aided simulations to optimize the cooling treatment in order to keep the residual stresses below a given admissible threshold and identify the required initial geometry of the glass piece so that after cooling, it matches precisely the desired one.

The case treated here corresponds to an optical device and the thermomechanical analysis is performed using the commercial Finite-Element code Ansys. The geometry and boundary conditions are shown on Figure 1. The glass piece has a symmetry of revolution and occupies the domain $\Omega$ bounded by the surface $\Gamma=\Gamma_{1} \cup \Gamma_{2} \cup \Gamma_{3} \cup \Gamma_{4}$ at $t=0$. It is assumed to be initially stressfree and with uniform temperature $T_{0}=873.15 \mathrm{~K}$. Radiative heat transfer is ignored so that the temperature field within the glass piece is dictated by the heat diffusion equation. Moreover, heat is lost to the surrounding through convective heat transfer characterized by a constant coefficient of heat transfer $h$. In this optimization problem, the time-dependent temperature of the surrounding $T_{a}(t)$ is the control used to minimize an objective function yet to be defined.

Upon cooling, the glass behavior undergoes drastic changes. At high temperature $\left(T>T_{g}+100 \mathrm{~K}\right)$ it behaves like a Newtonian liquid while at lower 


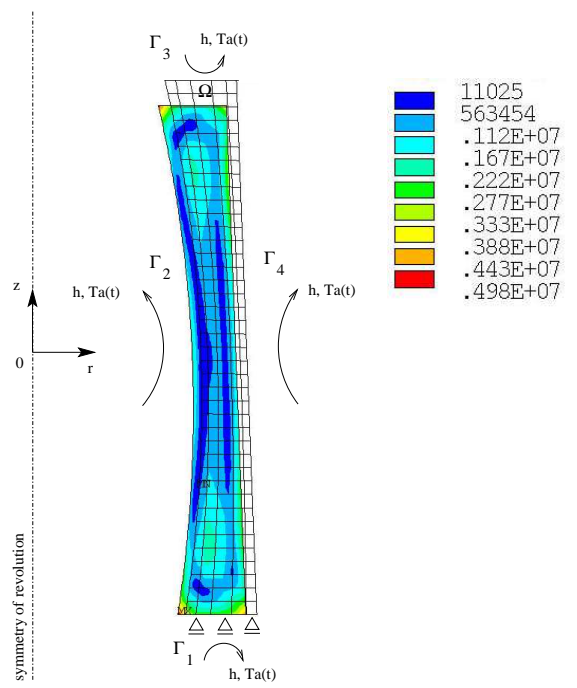

Fig. 1. Initial glass piece geometry with corresponding Finite Element mesh and boundary conditions. The deformed glass geometry (after cooling) with the associated map of the residual Von Mises stresses is also shown.

temperatures $\left(T<T_{g}-50 \mathrm{~K}\right)$, classical linear elasticity applies. In the intermediate temperature range, the glass is best described as a viscoelastic solid where stress and structure relaxation occur. The state of the structure of the glass is characterized by the fictive temperature $T_{f}(\mathbf{x}, t)$ a concept well established after the work of [Narayanaswamy (1971)]. Accordingly, constitutive laws may be expressed in the following integral form,

$$
\begin{aligned}
s_{i j}(\mathbf{x}, t) & =2 \int_{0}^{t} G\left(\xi(\mathbf{x}, t)-\xi\left(\mathbf{x}, t^{\prime}\right)\right) \frac{\partial e_{i j}\left(\mathbf{x}, t^{\prime}\right)}{\partial t^{\prime}} d t^{\prime} \\
\sigma(\mathbf{x}, t) & =3 K\left(\epsilon(\mathbf{x}, t)-\epsilon_{t h}(\mathbf{x}, t)\right)
\end{aligned}
$$

where $s_{i j}, \sigma$ and $e_{i j}, \epsilon$ are the deviatoric and volumetric parts of the stress and strain tensor respectively. The bulk modulus $K$ is chosen to be constant while the shear modulus $G$ is a function of the elapsed reduced time, $\xi(\mathbf{x}, t)-\xi\left(\mathbf{x}, t^{\prime}\right)$. A classical Arrhenius model is used to represent the influence of the temperature on the relaxation behavior so that the relaxation time is expressed as:

$$
\xi(\mathbf{x}, t)=\int_{0}^{t} \frac{\tau_{r e f}}{\tau\left(T, T_{f}, t^{\prime}\right)} d t^{\prime}=\int_{0}^{t} e^{\frac{\Delta H}{R}\left(\frac{1}{T_{0}}-\frac{\beta}{T\left(\mathbf{x}, t^{\prime}\right)}-\frac{1-\beta}{T_{f}\left(\mathbf{x}, t^{\prime}\right)}\right)} d t^{\prime}
$$

where $\tau_{\text {ref }}$ and $\tau\left(T, T_{f}, t^{\prime}\right)$ are the relaxation times at the initial temperature and the temperature $T$, respectively. $\beta$ is a constant $(0<\beta<1), \Delta H$ the activation energy and $R$ the ideal gas constant. The shear modulus and fictive temperature are expressed in the form of Prony series, viz 


$$
\begin{aligned}
G(\xi) & =G_{\infty}+\sum_{i=1}^{n} G_{i} e^{-\xi / \lambda_{i}} \text { with } G_{i}=\nu_{i}\left(G_{0}-G_{\infty}\right) \text { and } \sum_{i=1}^{n} \nu_{i}=1, \\
T_{f}(\mathbf{x}, t) & =\sum_{i=1}^{m} \omega_{i} T_{f_{i}}(\mathbf{x}, t) \text { with } \sum_{i=1}^{m} \omega_{i}=1 .
\end{aligned}
$$

In eqs. (4) and (5), $G_{0}$ and $G_{\infty}$ are the initial and final shear moduli, respectively, while $G_{i}$ and $\omega_{i}$ are weights and $\lambda_{i}$ are constants associated with a discrete relaxation spectrum in shear. $T_{f_{i}}$ are the partial fictive temperatures and these must satisfy the following ODE, [Markovsky et al. (1984)]:

$$
\frac{d T_{f_{i}}}{d t}=-\frac{T_{f_{i}}-T}{\mu_{i}} \frac{d \xi}{d t},
$$

where $\mu_{i}$ are constants associated with a discrete structural relaxation spectrum. Finally, the thermal strain in eq. (2) is given by:

$$
\epsilon_{t h}=\alpha_{g}\left(T-T_{0}\right)+\left(\alpha_{l}-\alpha_{g}\right)\left(T_{f}-T_{0}\right),
$$

where $\alpha_{g}$ and $\alpha_{l}$ are the coefficients of thermal expansion of solid and liquid glass respectively. The glass transition occurs around $T_{g}=773.15 \mathrm{~K}$.

The glass piece is assumed to be traction free and slides without friction on its base. Numerical simulations proceed by first computing the temperature field until the temperature in the glass is uniform and equal to the room temperature $(293.15 \mathrm{~K})$ and then impose it as a load to the structural analysis. A typical map of the residual Von Mises stresses can be seen on Figure 1.

\section{Optimization of the cooling curve}

A first step in the identification of the required initial shape consists in optimizing the cooling curve in order to reduce the permanent stresses produced by temperature gradients. To this end, the algorithm proposed by [Sonmez et al. (2002)] was employed which attempts to reduce the total cooling period while keeping residual stresses below a prescribed threshold, $\sigma_{\text {adm }}$.

As seen on Figure 2, three regions define the cooling curve. The stresses only have the ability to relax in the first region characterized by $T_{g}-50<$ $T_{a}<T_{g}+100$. The optimization is therefore restricted to this part of the cooling curve. In the region to be optimized, the cooling curve is defined by $N=7$ key-points with locations $\left(i \Delta t, T_{i}\right), i=1, \ldots, N$, where $\Delta t$ is a time interval. The initial and final temperatures are fixed to $T_{a}(t=0)=T_{0}$ and $T_{a}\left(t_{f}=(N+1) \Delta t\right)=723.15 K$ and the temperature is interpolated linearly between the key-points. The optimization problem has therefore $N+1$ degrees of freedom, namely the $N$ values of the temperature $T_{i}$ and $\Delta t$.

The aim of the optimization is to reduce the total cooling time $t_{f}$ subject to the constraint that the maximum value of the Von-Mises stresses should not 

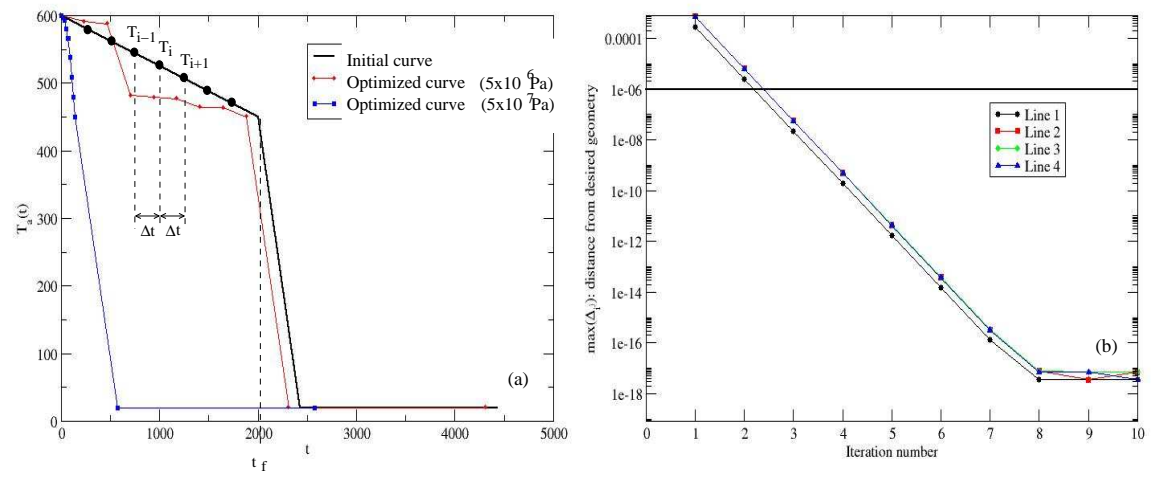

Fig. 2. (a): Initial and optimized cooling curves for $\sigma_{a d m}=5 \times 10^{6} \mathrm{~Pa}, \sigma_{a d m}=$ $5 \times 10^{7} \mathrm{~Pa}$; (b): Convergence history of the proposed algorithm for each line $\Gamma_{i}$.

exceed $\sigma_{a d m}$ at room temperature. Moreover, realistic cooling curves should be monotonically decreasing and the slope bounded by a constant $\kappa=1 \mathrm{~K} / \mathrm{s}$ in order to avoid exceedingly large temporary stresses. Accordingly, the optimization problem is formulated as follows:

$$
\min _{T_{i}, \Delta t} t_{f}\left(1+P_{1}+P_{2}+P_{3}\right)
$$

where the $P_{i}$ correspond to the penalty functions associated with each constraint. These are given by:

$$
\begin{aligned}
& P_{1}= \begin{cases}C_{1} \frac{\max _{\mathbf{x} \in \Omega}\left(\sigma_{V M}\left(\mathbf{x}, t=t_{e}\right)\right)-\sigma_{a d m}}{\sigma_{a d m}} & \text { if } \max _{\mathbf{x} \in \Omega}\left(\sigma_{V M}\left(\mathbf{x}, t=t_{e}\right)\right)>\sigma_{a d m} \\
0 & \text { if } \max _{\mathbf{x} \in \Omega}\left(\sigma_{V M}\left(\mathbf{x}, t=t_{e}\right)\right) \leq \sigma_{a d m}\end{cases} \\
& P_{2}=\left\{\begin{array}{ll}
C_{1} \frac{T_{i+1}-T_{i}}{T_{0}} & \text { if } T_{i+1}>T_{i} \\
0 & \text { if } T_{i+1} \leq T_{i}
\end{array}, P_{3}= \begin{cases}C_{1} \frac{T_{i+1}-T_{i}}{\kappa \Delta t} & \text { if }\left\|T_{i+1}-T_{i}\right\|>\kappa \Delta t \\
0 & \text { if }\left\|T_{i+1}-T_{i}\right\| \leq \kappa \Delta t\end{cases} \right.
\end{aligned}
$$

The constant $C_{1}$ is chosen to be equal to $10^{6}$. The Nelder-Mead simplex direct search method from Matlab was adopted to minimize eq. (8).

The initial and optimized cooling curves for $\sigma_{a d m}=5 \times 10^{6} \mathrm{~Pa}$ and $\sigma_{a d m}=5 \times 10^{7} \mathrm{~Pa}$ are shown on Figure 2 (a). As expected when the constraint on the maximum admissible Von Mises stress is least severe, a much quicker cooling is possible. The shape of the cooling curve for $\sigma_{a d m}=5 \times 10^{6} \mathrm{~Pa}$ is also as expected: after an initial rapid cooling, the temperature remains approximately constant. This feature allows the stresses to relax to the admissible level. Finally, the map of the residual Von Mises stresses when $\sigma_{a d m}=5 \times 10^{6}$ Pa shown on Figure 1 confirms that, the level of stress is kept below $\sigma_{a d m}$. 


\section{Identification of the required initial geometry}

In order to describe the algorithm which tackles the inverse problem of identifying the required initial geometry, notations are introduced. Let $M_{1}^{d}, \ldots, M_{L}^{d}$ denotes the $L$ boundary nodes of the desired glass geometry at room temperature. The required initial geometry is found by updating the location of the boundary nodes $M_{i}^{j}$ iteratively (the superscript indicates the iteration number). At each iteration $N_{i}^{j}$ corresponds to new location of the node $M_{i}^{j}$ in the

deformed geometry and $\vec{U}_{i}^{j}$ is the associated displacement. The algorithm is defined in pseudo-code notation as follows:

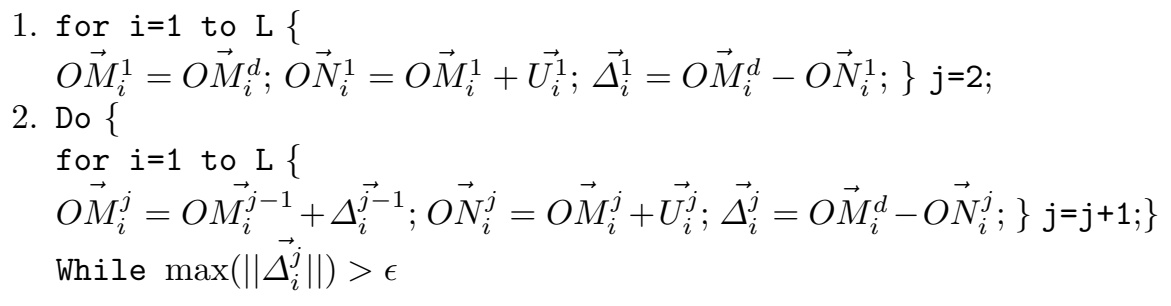

Stated in simpler terms, the initial guess for the required initial boundary node locations is taken to be the location of the nodes of the desired geometry at room temperature. At each iteration the residual vector $\left(\overrightarrow{\Delta_{i}^{j}}\right)$ which measures how far the deformed geometry is from the desired one is evaluated and added to the previous guess of the required initial boundary node location.

This algorithm was tested for the case when the desired geometry at room temperature corresponds to the initial geometry on Figure 1 and the cooling treatment is as shown on Figure 2 (a) with $\sigma_{a d m}=5 \times 10^{6} \mathrm{~Pa}$. The convergence history is displayed on Figure 2 (b). For each of the $\Gamma_{i}$ of the contour $\Gamma$, the maximum of the Euclidean norm of $\vec{\Delta}_{i}^{j}$ is plotted against the number of iteration. The convergence rate is around two decades per iteration which is very satisfactory and the Micron threshold is achieved after three iterations.

Acknowledgement. The author gratefully acknowledges the funding of the European Union through the MAGICAL project.

\section{References}

[Narayanaswamy (1971)] Narayanaswamy, O.S.: A model of structural relaxation in tempering glass. J. Am. Ceram. Soc. 54(10), 491-498 (1971)

[Markovsky et al. (1984)] Markovsky, A., Soules, T.F.: An efficient and stable algorithm for calculating fictive temperatures. C. Am. Ceram. Soc. 67, C-56-C-57 (1984)

[Sonmez et al. (2002)] Sonmez, F.O., Eyol, E.: Optimal post-manufacturing cooling paths for thermoplastic composites. Composites: Part A 33, 301-314 (2002) 